\title{
Pradoluengo y Paderluengo. ¿Un híbrido vascorrománico camuflado? Pradoluengo and Paderluengo. A hidden Romano-Basque hybrid?
}

\author{
David Peterson \\ Euskal Herriko Unibertsitatea UPV/EHU
}

\begin{abstract}
$^{1}$
Pradoluengo would seem to be one of the most transparently Romance place-names in the province of Burgos, from the Latin etyma pratum and longus. However, all the medieval references to the town, from various sources, insist on quite different forms -essentially Paderluengo - which it is difficult to derive from pratum, obliging us to consider alternatives. Within the context of the Sierra de la Demanda, an area teeming with Basque place-names, a Basque origin seems a plausible alternative, and in fact the name would appear to be a hybrid, formed from Basque padur(a) 'water-meadow' and Latin longus. Hybrids of this type are useful in that they allow us to understand and potentially even contextualise in chronological terms the period of Basque-Romance bilingualism in this area.
\end{abstract}

Keywords: hybrid, bilingualism, Basque, Romance, place-names, Pradoluengo, early medieval.

\section{Resumen}

Pradoluengo parece ser uno de los topónimos más transparentes de toda la provincia de Burgos, a partir de los étimos latinos pratum y longus. Sin embargo, las referencias medievales a la villa en diversas fuentes insisten en unas formas - esencialmente Paderluengo- que difícilmente se deriven de pratum, lo cual nos obliga a contemplar etimologias alternativas. En el contexto de la Sierra de la Demanda, una comarca profusa en toponimia vasca, un origen euskérico es una posibilidad a tener en cuenta, y parece verosimil que en realidad se trata de un topónimo hibrido vascorrománico, compuesto del

${ }^{1}$ Este trabajo se ha realizado dentro del marco del proyecto de investigación «Escribir el espacio en la alta Edad Media: una aproximación comparada a la relación entre escritura y acceso a la tierra» (HAR2013-44576-P), financiado por el Ministerio de Ciencia e Innovación. Quiero agradecer la ayuda de la profesora Emiliana Ramos en la preparación de este trabajo. 
étimo vasco padur(a) 'vega' y el latino longus. Este tipo de híbrido nos ayuda a entender e incluso contextualizar cronológicamente un periodo de bilingüismo vascorrománico en la comarca.

Palabras clave: hibrido, bilingüismo, vascorrománico, Pradoluengo, toponimia vasca, altomedievo.

Pradoluengo es una villa burgalesa situada en la estribaciones septentrionales de la Sierra de la Demanda. Su nombre, a primera vista, parece ser uno de los más transparentes de toda la provincia, a partir de los étimos latinos y después romances pratum y longus. Tampoco desentona la diáfana etimología resultante de 'prado alargado' en el contexto de un estrecho valle inserto en un espacio de gran tradición ganadera. Si esta realidad topográfica está ahora algo sepultada por el posterior éxito industrial del núcleo y la consecuente urbanización del fondo del valle, esto de nuevo se debe a la feliz coyuntura de un valle estrecho con un aprovechable corriente de agua en un espacio idóneo para la ganadería lanar. En resumen, la topografía parece apoyar la etimología obvia, y si a esto se añade el hecho de que ninguno de los dos étimos romances contemplados (pratum y longus) desentone en la toponimia de la región, ${ }^{2}$ se consolida aún más una etimología ampliamente aceptada.

El problema surge cuando se consulta la documentación medieval. Una etimología que parecía obvia y topográficamente coherente choca con las formas adoptadas por el topónimo en la documentación medieval: Paterlongo y Paderlongo en el siglo XI, y después Padrelongo en los siglos XIII-XIV. Evidentemente el segundo elemento (longo < lat. longus) no presenta ningún problema, pero las formas primitivas Pater o Pader, así como su heredero bajomedieval Padre, muy difícilmente se hacen derivar de lat. pratum. Si antes hemos comentado la gran acepción de este étimo latino en la toponimia del entorno, es llamativa también su inalterabilidad morfológica en esas múltiples fosilizaciones toponímicas, al margen de la sonorización de la con-

\footnotetext{
2 Para pratum los ejemplos microtoponímicos son innumerables, pero restringiéndonos a la macrotoponimia más inmediata tenemos Pradilla, unos siete kilómetros al este en línea recta. El adjetivo longus ha prosperado menos en la toponimia mayor, pero está representado en Solduengo (primitivamente Soto Longo BGD \#230, 1003) en la Bureba, 35 kilómetros al norte. En referencia a las diferentes fuentes medievales que se manejan en este trabajo se emplean las siguientes abreviaturas:

- BGD = Becerro Galicano Digital [de San Millán de la Cogolla] http://www.ehu.es/galicano, consultado 11/212/2014;

- Bujedo = El Libro Becerro de Santa María de Bujedo de Candepajares (1168-1240), ed. S. Ruiz De Loizaga, Miranda de Ebro, 2000;

- Cardeña = Colección documental del Monasterio de San Pedro de Cardeña, ed. G. Martínez Díez, Burgos, 1998;

- Froncea = Cartulario de San Miguel de Froncea (inédito), Biblioteca de la Univ. de Oviedo (sign \#456) - manejamos una transcripción preparada por la profesora Josefa Sanz;

- Oña = Colección Diplomática de San Salvador de Oña (822-1284), ed. J. del Alamo, Madrid, 1950;

- Valvanera = Documentación medieval del monasterio de Valvanera, siglos XI a XIII, ed. Francisco Javier García Turza, Zaragoza, 1985.
} 
sonante interna $(T>d)$ y algún caso de variación de género; ${ }^{3}$ y ahora es precisamente esa facilidad con que pratum se asimila inalteradamente en la toponimia el mayor argumento en contra de que Pater / Pader haya derivado de ello.

A esto debemos añadir que la problemática forma no aparece una sola vez ni en una sola fuente, sino en diferentes fuentes no interrelacionadas entre sí, pero cuyos testimonios en este punto coinciden. Para ilustrar esto, antes de explorar posibles explicaciones para la problemática forma primitiva, conviene examinar las menciones del asentamiento en la documentación medieval.

\section{Pradoluengo en la documentación medieval}

1. Las primeras dos referencias a la villa de Pradoluengo aparecen en dos redacciones diferentes — aunque firmadas por el mismo amanuense (Tello $\mathrm{Mu}$ ñoz) - de un texto de 1090, ambas copiadas en el Becerro Galicano emilianense pero provenientes originalmente del monasterio de San Miguel de Pedroso (convertido en priorato de San Millán a mediados del siglo XI):

- terra inter Villagalisso et Paderluengo in illis padulibus (BGD332, fol. 98v);

- terra inter Villa Alisso et Paderlongo innas padules (BGD353, fol. 107).

Observamos la perfecta contextualización comarcal (Villagalijo se sitúa tres kilómetros al norte de Pradoluengo), a lo cual podemos añadir que el origen del texto fue San Miguel, en sí apenas ocho kilómetros al norte y el centro jerarquizante de toda la comarca. Si añadimos el hecho de que hayan sobrevivido dos redacciones del texto, no puede quedar ninguna duda de que, al margen de la diptongación de la o > ue, la forma del topónimo en 1090 fuera efectivamente Paderlongo. En otras palabras, esta forma no es el resultado de una errata, producida ni por lapsus calami ni por desconocimiento del entorno. Antes de abandonar el ejemplo, notemos también la voluntad latinizante del escribano de la versión en el folio 98v (innas padules > illis padulibus), quien no obstante, no corrige Pader- a Prato- o Prado-. También notemos, para posterior referencia, el género femenino de padules.

2. Las tres siguientes apariciones del nombre, todas ellas en un mismo texto del año 1095 (BGD653), también se han conservado en el Becerro Galicano de San Millán, pero ahora en otra parte del códice que desvela una transmisión archivística diferente a la de las dos versiones del texto de 1090. Aparece hacia el final del códice, en su folio 210 , fuera ya de la estructura geográfica imperante en la primera parte del cartulario, en una sección estructurada, en cambio, alrededor de la memoria de los individuos donantes. En esta ocasión no disponemos

3 Ejemplos tomados de diferentes fuentes de la incorporación inalterada del étimo pratum en la topografía y toponimia del entorno de Pradoluengo: illo prato de Lazar (Oña27, 1035); Pratiella (BGD472, 1044); pratello de Sorripa (Cardeña265, 1050); Prato (Cardeña293, 1062); Pratiella (Valvanera46, 1068); Pratielo (BGD252, 1076); Prato Mediano (BGD109, 1086); Prato (BGD272, 1091); pratum de Spina (Oña107, 1096); barrium quod dicitur de Prato (Oña259, 1177); Prato (Froncea53, 1202); Prado longo (Bujedo131, 1209); Pradiello (Bujedo139, 1211). 
del nombre del escribano, ni tampoco de una referencia explícita a su factura en el priorato de San Miguel de Pedroso, pero la inclusión en la lista de testigos de diversos representantes del entorno inmediato de Pradoluengo (Valle de San Vicente, Villagalijo, Fresneda) de nuevo indica una redacción local. A las dos referencias del cuerpo del texto podemos añadir la rúbrica, plausiblemente la obra del monje que hacia 1115 recopiló la documentación para el perdido Becerro Gótico que parece haber servido de fuente para el Becerro Galicano. De todos modos, al margen de la falta de sonorización $t>d$, la forma del topónimo, repetido tres veces, es consistente con lo que hemos visto arriba:

- De divisa in Paterlongo cum solare (BGD653, fol. 210 - en rúbrica)

- in Paterlongo, uno solare cum sua divisa (BGD653, fol. 210)

- et concilium de Paterluengo, testis (BGD653, fol. 210).

3. Para la siguiente aparición del nombre debemos esperar hasta mediados del siglo XIII. No obstante, el ejemplo es valioso porque nos aleja del archivo de San Millán, cuando vemos cómo en el inédito Cartulario de Froncea ${ }^{4}$ el nombre del pueblo, de nuevo en un contexto claramente demandés, ${ }^{5}$ se ha convertido en Padreluengo: De Padreluengo: Pascual e Pedro Ezquiedo (Froncea105, 1254). Si bien supone un cambio desde la forma vista a finales del siglo XI, se trata de un cambio asumible dentro de la fonética, con toda probabilidad influenciado por el análogo cambio en el léxico común de pater > padre, y que nos deja una forma igualmente problemática hacer derivar del putativo pero inmutable étimo pratum. En otras palabras, todo indica que el Padre- del siglo XII derivase del Pader- del siglo XI.

4. Por último, toda la sierra de la Demanda es destacada en el Libro de Montería (LM) de Alfonso XI por la excelencia de sus montes para la caza del oso, y los folios 97 y 98 de esta fuente del siglo XIV nos proporcionan otras tres menciones de la villa serrana. ${ }^{6}$ Consistente con lo que hemos visto en Froncea, encontramos de nuevo la forma Padre Longo en dos ocasiones, y en otra ocasión Had Luengo, lo que sí parece ser una errata, quizás influenciada por la referencia al hayedo (Haedo) que precede los otros ejemplos. La estructura geográfica de la obra no deja lugar a dudas de que se está hablando de nuevo de nuestro Pradoluengo, y por si acaso disponemos también de una referencia al pueblo vecino de Santa Cruz (tres kilómetros al oeste):

- el Haedo de Padre Longo et Monte Agudo es todo un monte (LM, fol. 97)

- entre el Haedo de Padre Longo et la Garganta de Sancta Cruz (LM, fol. 97)

- Val de Cabrones, et Had Luengo es todo un monte (LM, fol. 98).

\footnotetext{
${ }^{4}$ Es un cartulario del siglo XIV que contiene textos de los siglos X a XIII. Manejamos una transcripción preparada por la profesora Josefa Sanz con visos a su futura edición. Actualmente, el cartulario se halla en la Biblioteca de la Universidad de Oviedo, y el fragmento que citamos a continuación se halla en su fol. 30r ${ }^{\circ}$.

5 En la lista de testigos, además de Pradoluengo, aparecen referencias a Alarcia, Arlanzón, Herramel, Pineda, el Valle de San Vicente y Villorobe.

6 Seniff, Dennis P. (ed.), Alfonso XI. "Libro de la montería." Based on Escorial MS Y.II.19. Madison: Hispanic Seminary of Medieval Studies, 1983.
} 
Para complementar esta nómina de referencias tempranas a Pradoluengo, ahora pasaremos a contemplar otros dos casos que tradicionalmente se han considerado referentes a la villa. Aunque ahora sabemos que no es así, nos aportan datos valiosos sobre el comportamiento toponímico de diferentes voces que nos interesan.

El primer caso aparece en otro texto procedente del Becerro Galicano de San Millán, en el cual aparece la secuencia de alia pars Heterna latus Paterlongo (BGD392, 1084). Tradicionalmente se ha considerado ésta como la primera mención del pueblo, pero en realidad se refiere al entorno de Cerezo unos veinte kilómetros al norte. ${ }^{7}$ Pero, lejos de ser una irrelevancia por no referirse a nuestro Pradoluengo, el hecho de que pader (aquí pater) y longo coincidan una segunda vez en una construcción toponímica sugiere que dicha combinación no es fortuita o errónea, sino que la voz pader, sea cuál sea su significado, fuera un elemento vivo del léxico comarcal de finales del siglo XI.

El segundo caso anómalo pero instructivo es la aparición en el Fuero de Cerezo del topónimo Pradolongo, y que también se ha entendido tradicionalmente como una referencia a la villa serrana que nos ocupa. ${ }^{8}$ El texto del fuero incorpora una fecha de 1151, pero sólo nos es conocido a partir de la copia hecha por Llorente hacia 1807 y que contiene anacronismos manifiestos, como sería la mención de San Juan de Ortega como asentamiento. ${ }^{9}$ El fuero se estructura geográficamente: primero una lista de pueblos de los Montes de Oca, luego los del alto Tirón, la Riojilla, el entorno de Belorado, la Bureba, el valle del río Oca, los Montes Obarenes, el curso inferior del Tirón, y a final varios pueblos de la Cuenca de Miranda y Valdegobía. En esta rigurosa estructura espacial sólo hay un asentamiento que parece desentonar: el que lleva el nombre Pradolongo, ${ }^{10}$ alejado de los asentamientos del alto Tirón y situado entre Treviana y Pancorbo, unos 32 kilómetros al noreste. Pero, lejos de estar 'fuera de lugar', este Pradolongo está perfectamente ubicado, pues no hace referencia a nuestro Pradoluengo sino a una pequeña aldea altomedieval, situada entre Treviana y Pancorbo ${ }^{11}$ y llamada Paduluenga (BGD596, 1128), Padul longa (BGD180, 1088) o Pauleja en la documentación medieval, ésta última la forma dominante aunque no por ello necesariamente la original. ${ }^{12}$ Hoy abandonado el lugar, el primitivo asentamiento es recordado en el hidrónimo Pradoluenga (cerca de Villaseca) cuya discordancia de género desvela el origen del primer elemento en padul < lat. palus, paludis y no pratum. La similitud de esta forma con el nombre de la villa burgalesa que nos ocupa es llamativa y explicaría cómo,

${ }^{7}$ La toponimia (tanto micro- como macro-) del documento no deja lugar a dudas: Summas Ventanas (Quintanilla de las Dueñas, Cerezo), Valle de Fratres (Valdefrades, Cerezo), Palatio (Los Palacios, Cerezo), Arto (río Arto), costa de civitate (Campo Ciudad, Cerezo), Carrasketo (Carrasquedo, Redecilla del Campo), Ibrielos (Ibrillos), Sancti Mames (ermita de Redecilla del Campo), Tolmantos (Tormantos), VII Finiestras (Quintanilla de las Dueñas, Cerezo), Quintaniella (Quintanilla de las Dueñas, Cerezo).

8 ed. G. Martínez Díez, Fueros Locales en el territorio de la provincia de Burgos, 1982.

${ }_{9}^{9}$ El religioso que se convertiría en santo no murió hasta 1163 , mientras el asentamiento hospitalario que fundó, primitivamente conocido como de San Nicolás, no llevaría su nombre hasta el siglo XIII.

$10 «$ El único caso que se encuentra fuera de lugar es el de Pradolongo, identificado con Pradoluengo, que debería estar incluido con los pueblos del valle alto del Tirón, y que aparece en solitario, tras los del valle bajo del Tirón», Pérez Avellaneda, Cerezo de Río Tirón, p. 187.

${ }_{11}$ Como la propia secuencia del fuero indica: Quintanilla de las Duennas, Arto, Trepeana, Pradolongo, Pancorvo, Encio, Ameiucus [por Ameyugo].

12 Padulella (BGD1776, 1070), Paduleia (BGD166, 1095), y Padulegga (BGD20, 1098; BGD736, 1163; BGD178, 1168). 
en algún momento indeterminado del bajomedievo —o incluso más tarde — cuando se hizo la copia del fuero que conocemos y que incorpora la referencia a San Juan de Ortega, con la aldea altorriojana ya abandonada y medio olvidada, se cambió el hipotético Paduluenga o Pradoluenga en Pradolongo.

En resumen, en diferentes fuentes a lo largo de tres siglos, todas las referencias a Pradoluengo nos alejan del, por lo demás inalterable, étimo pratum y nos indican que la etimología original fuera otra. Las formas que presenta el nombre no son el resultado de una serie de erratas aleatorias, pues son coherentes entre sí, ni de desconocimiento del entorno, y tampoco parecen ser el resultado de contaminación textual al aparecer en fuente diversas. ¿Cómo, entonces, explicar estas formas?

- Paderluengo (1090, BGD332)

- Paderlongo (1090, BGD353)

- Paterlongo (1095, BGD653)

- Paterluengo (1095, BGD653)

- Paterlongo (1095, BGD653 [rúbrica])

- Padreluengo (1254, Froncea105)

- Padre Longo (s. XIV, Libro de Montería)

- Padre Longo (s. XIV, Libro de Montería)

- Had Luengo (s. XIV, Libro de Montería)

\section{La toponimia vasca de la Sierra de la Demanda}

Lo que no hemos mencionado hasta ahora es que la comarca de Pradoluengo dista de ser toponímicamente monolingüe, pues la villa se sitúa dentro de la zona de mayor concentración de toponimia vasca fuera del actual Pais Vasco o Navarra. Son centenares los topónimos vascos del entorno, estudiados primero por Merino Urrutia, ${ }^{13}$ y más recientemente por otros como Mujica Urdangarín ${ }^{14}$ o Gómez Villar, ${ }^{15}$ y cuyo origen parece remontar al altomedievo (Peterson). ${ }^{16}$ En Pradoluengo también abundan topónimos vascos (Basardia, Bizkarra, Gutillarana, Ordokia, Regoluna, Remendia, Zarracaturria ...), de los cuales Mujika recoge unos 200 ejemplos más o menos claros. En este contexto, y a pesar de la transparencia romance del segundo elemento de Paderluengo, parece lógico por lo menos contemplar que el léxico o la fonética vascos hayan influido en el desarrollo del topónimo problemático.

Para el primer elemento de Padregutia, un microtopónimo de Valgañón en el cercano valle de Ezcaray con un segundo elemente claramente vasco, Henrike Knörr propuso la etimología padre $\left[<{ }^{*}\right.$ pader? $]<$ padura < bajo lat. padul < lat. palus, paludis..$^{17} \mathrm{En}$ euskera el significado de padura habría evolucionado desde el significado original de 'la-

\footnotetext{
13 Merino Urrutia, Jose J. Bautista, La Lengua Vasca en La Rioja y Burgos, Logroño, 1978.

14 Mújika, Luis María, "Toponimia vasca en Burgos (nuevos testimonios)", $11^{\circ}$ Congreso de Estudios Vascos: San Sebastián (1991), 423-433.

15 Gómez Villar, Rufino, «Toponimia vasca en la comarca de Belorado (Burgos)», FLV 92 (2003), 179-197.

16 Peterson, David, Frontera y Lengua en el Alto Ebro (ss. VIII-XI), Logroño, 2009.

17 Knörr, Henrike, «El euskera en tierras del romance: Rioja Alavesa, La Rioja, Burgos, Encartaciones», en Vascuence y Romance: Ebro - Garona. Un espacio de comunicación: Pamplona (2004), 41-50.
} 
guna, humedal' del latino palus, para extenderse a la tierra próxima al metonímico humedal y Michelena sugiere 'vega' como una segunda acepción, ${ }^{18}$ propuesta acogida por Knörr en referencia a Padregutia, que así significaría 'vega pequeña'. ${ }^{19}$

Padura es un elemento muy productivo en la toponimia vasca, estudiado primero por Knörr y más recientemente por Zuazo como uno de media docena de marcadores diferenciales entre los dos dialectos primitivos del euskera alavés. ${ }^{20}$ Así la toponimia de Álava nos aporta ejemplos interesantes que documentan el progresivo adelantamiento de la $r$ en algunos topónimos: Padrugucheta en Eribe, o Padruquelu en Oreitia, y Praduzuya en Urarte. ${ }^{21}$ Conviene puntualizar que el recurso a ejemplos alaveses no es gratuito dada la contrastada similitud entre el euskera demandés y el alavés, observable ya en la documentación medieval con las formas occidentales -uri y baltza, ${ }^{22}$ y ampliamente comentada por todos los estudiosos del tema. ${ }^{23}$

No obstante, hay una clara diferencia entre Padregutia y Paderluengo: el elemento adjetival es vasco en Ezcaray, pero romance en Pradoluengo. Si aceptamos que $p a-$ der / padre, a pesar de su remoto origen latino, es aquí un vasquismo, ¿es factible este tipo de híbrido vascorrománico?

Parece probable que el topónimo burgalés Solduengo recorriera un camino parecido, pues a pesar de la forma medieval y quizás culta Sotoluengo (Cogolla123, 1003), tanto la $l$ como la $d$ actuales se explican mejor a partir del vasco zaldu que del equivalente romance soto, que no conserva rastro alguno de la l latina en la toponimia romance medieval. ${ }^{24}$ Ambas voces derivan del latín saltus, pero sólo en vasco se preserva la $l$, asimismo la sonorización de la oclusiva $(t>d$, lat. saltus $>$ zaldu) es también característica del euskera. Hay dos explicaciones posibles: la vasconización parcial de un topónimo puramente romance (Soto luengo) después de su aparición en 1003; o la latinización más o menos culta (y quizás únicamente en el registro escrito, puesto que la forma actual del topónimo atestigua la supervivencia de la forma vasca en el registro popular) de un híbrido vascorrománico. Nos parece más probable la segunda solución, ya que la dinámica de castellanización de topónimos vascos es un hecho

18 Michelena, Luis, "Apellidos vascos», en Obras Completas, IX Onomástica, eds. J. A. Lakarra \& Í. Ruiz Arzalluz, San Sebastián y Vitoria, 2011, 1-236, aquí p. 136 (\#514).

19 Otra posible aparición de padura de la documentación medieval referente a la Sierra de la Demanda es el nombre Paderey (Valvanera205, 1110).

20 Knörr, "Para una delimitación etno-lingüística de la Álava antigua. Ensayo de cartografía a partir de pruebas toponímicas", en La Formación de Alava: Vitoria-Gasteiz (1984), 489-541. Zuazo, Koldo, El Euskera de Álava, Vitoria-Gasteiz, 2013, 15.

21 Knörr, «Para una delimitación etno-lingüística ...», 511.

22 - uri es más frecuente en el curso inferior del río Tirón con los topónimos actuales Cihuri, Herramélluri, Ochánduri todos ellos profusamente documentados en el Becerro Galicano (por ejemplo, BGD419, 1111), pero también observable aguas arriba, cf. Villagariguri (BGD13*, 1129), en referencia al ya mencionado Villagalijo. Por su parte, baltza aparece en el topónimo Valle de Balza (BGD303, 1049), en San Miguel de Pedroso.

23 Alarcos Llorach, Emilio, "Apuntaciones sobre toponimia riojana", Berceo 16 (1950), 492; Michelena, "Onomástica y Población en el antiguo reino de Navarra» (1976) y "Sobre la lengua vasca en Alava durante la Edad Media» (1982), ambos en Obras Completas V.

24 Ejemplos del romance soto (< lat. saltus) sin indicios de la $l$ latina en la documentación emilianense: Soto (BGD340, 979), Sauto / Soto (BGD701, 1028), Saucto (BGD351, 1037), Saucto (BGD352, 1045), Sotello (BGD402, 1073), Sotiello (BGD160, 1102), Soto (BGD255, 1108), Sotello (BGD403, 1109), Sotello (BGD404, 1109), Soto (BGD732, 1116). 
ampliamente observado en espacios de contacto entre las dos lenguas, ${ }^{25}$ mientras la dinámica inversa de vasconización parcial parece mucho menos verosímil.

¿Cómo deberíamos considerar el étimo padre, cómo un vasquismo propiamente dicho o cómo una voz romance que haya sido alterada fonéticamente? ¿No podría ser $\mathrm{Pa}$ derlongo simplemente un derivado a partir de padul + longus sin intervención vasca, solución que nos evitaría el problema del hibridismo? Pesan varios argumentos en contra de esta solución. En tal caso se esperaría la pérdida de la $d$ intervocálica, como ha ocurrido en los numerosos microtopónimos castellanos de tipo Paúl, Paúles, Pauleja etc. Una evolución exclusivamente romance tampoco explicaría la aparición de la $r$. Se esperaría, además, que se hubiera mantenido el género femenino que corresponde al sustantivo original, tal y como fue el caso con el Padul longa (BGD180, 1088) entre Treviana y Pancorbo que confundió al copista bajomedieval del Fuero de Cerezo y que todavía hoy se recuerda hidronímicamente como Pradoluenga. Y, por último, semánticamente el significado de 'vega' que Michelena atribuye a padura con toda probabilidad coincidiría mejor con la topografía preurbana del valle que el significado romance de 'laguna, humedal', si tenemos en cuenta la pronunciada pendiente que después hizo que el pueblo fuera tan apto para el desarrollo de la industria textil.

El resultado final supone un largo viaje desde el latino palus, paludis vía padul, padur, pader y padre hasta prado, y quizás parezca innecesariamente complejo, sobre todo si se tiene en cuenta que el postulado significado primitivo 'vega larga' es prácticamente el mismo que el significado moderno 'prado largo'. Sin embargo, por muy compleja que parezca la cadena, prácticamente todos sus eslabones están documentados. Tampoco suponen contravención alguna de las reglas fonéticas, salvo el último paso de $p a$ der > prado que se debería al bien conocido fenómeno de la etimología popular. Pero, sobre todo, una solución compleja es preferible a otra altamente improbable para no decir imposible, como sería la de pratum > pader > prado, que contradice el tan bien documentado y regular comportamiento toponímico de pratum en todos los demás casos observados. Si los significados son cercanos es precisamente porque describen una misma realidad topográfica: la orografía del valle. Lo que es paradigmático es que en el altomedievo se recurriera a una voz influenciada por la fonética vasca para hacer esto, mientras que unos siglos más tarde, con el significado de pader ya olvidado, se recurriera al étimo romance prado. Por lo tanto, este ejemplo retrata y hasta cierto punto contextualiza cronológicamente primero la vitalidad y después la decadencia del sustrato vasco en la Demanda y su posible influencia en el léxico local.

Por último, debemos contemplar las implicaciones sociolingüísticas de este tipo de topónimo híbrido. En la comarca es indudable la presencia de toponimia vasca, tanto en la actualidad como en la documentación medieval, y en este sentido el descubrimiento de un posible caso más entre centenares aporta poco. Observamos la toponimia vasca en la Sierra de la Demanda en gran abundancia y en diferentes estadios. En la mayoría de los casos sólo disponemos del testimonio de la toponimia actual, lo

25 Casi todos los autores que se han acercado al tema comentan ejemplos de castellanización de topónimos vascos en Burgos. Algunos de los casos más diáfonos y frecuentes son la consideración de una La- inicial en, por ejemplo, Larrea o Larramendi como el artículo definido romance, dejando formas como La Rea y Remendia; o la pérdida de la $i$ inicial en compuestos con iturri para dar Turrioza, Tordeluna de unos hipotéticos Iturrioza e Iturri-iluna. 
cual limita enormemente las posibilidades analíticas de contextualización cronológica, aunque algunas formas delatan la influencia del romance (Regoluna $<{ }^{*}$ Erreka Iluna, Remendia < * Larremendia y Turrioza < * Iturrioza), e híbridos como Molinazar o Bolincalze $e^{26}$ sugieren una población bilingüe en el (desconocido) momento del nombramiento. En este contexto las escasas pistas que nos ofrece la documentación medieval resultan preciosas por su capacidad de aproximarnos a la cronología de esa fase de bilingüismo. Es el caso de la convivencia de topónimos traducidos (Elhorzaha - Espinosa, Lizarraga - Fresneda) ${ }^{27}$ o la aparición de híbridos tautológicos (Fuente Lamiturri, BGD318, 945), que parecen indicar respectivamente conocimiento de las dos lenguas o desconocimiento de una de ellas (aunque quizás sólo por parte del escribano). El valor del posible híbrido Padreluengo es que complementa estos ejemplos, matizando la situación sociolingüística al documentar una fase de convivencia bilingüística: cuando una fértil ribera, lo que en alavés sería padura, pero pader en el dialecto local, se calificaba como luengo. No Padurluzea, que habría sido menos interesante, sino Paderluengo, y además no en un solo lugar, sino también veinte kilómetros aguas abajo del actual Pradoluengo donde se observa la misma forma en 1084. La repetición del híbrido sugiere que el vasquismo pader había sido plenamente absorbido en el dialecto romance comarcal en algún momento antes de las últimas décadas del siglo XI.

David Peterson

Universidad de Burgos

Facultad de Humanidades y Comunicacion

Paseo de Comendadores, s/n (Hospital Militar)

09001 Burgos, España

+34 618168305

dpeterson@ubu.es

\footnotetext{
${ }^{26}$ Elorza, Sanz Elorza, Mario, «Toponomástica molinológica de la provincia de Burgos», Revista de Folklore, 361 (2012), 4-33.

27 Elhorzaha (BGD13*, 1129) y Espinosa (BGD328, 945); Lizarraga (BGD307, 1028) y Fresneda (BGD340, 979).
} 